\title{
Coherent Optical System with Two-Amplitude Minimum-Shift Keying Format
}

\author{
Linglian Ouyang ${ }^{1, \mathrm{a}}$, Jing $\mathrm{He}^{1, \mathrm{~b}}$, Ming Chen ${ }^{1}, \mathrm{Jin}_{\mathrm{Tang}}{ }^{1}$, and Lin Chen ${ }^{1}$
}

${ }^{1}$ China Key Laboratory for Micro/Nano Optoelectronic Devices of Ministry of Education College of Computer Science and Electronic Engineering, Hunan University, Changsha 410082,

China.

a158359850@qq.com , bhnu_jhe@hotmail.com

Keywords: 2AMSK modulation; Optical coherent detection; SSMF

\begin{abstract}
In the paper, a digital optical communication system based on optical two-amplitude minimum shift keying (2AMSK) signal transmission with coherent detection is proposed. The simulation results show that, 100-Gb/s optical 2AMSK signal is successfully transmitted over $480 \mathrm{~km}$ standard single mode fiber (SSMF) with dispersion compensation.
\end{abstract}

\section{Introduction}

With the growing volume of network traffic, network bandwidth capacity requirements continue to increase. Recently, to further increase the system capacity and achieve high speed large capacity optical signals of long distance transmission, multi-dimensional multistage modulation format of high spectrum efficiency transmission technology research has been paid more attention. Advanced multi-level optical modulation formats, such as differential quadrature phase-shift keying (DQPSK), quaternary combined amplitude and differential quadrature phase-shift keying (ASK-DPSK) and 8-ary combined amplitude and differential quadrature phase-shift keying (ASK-DQPSK), have attached lots of research attentions because of greater spectral efficiencies and chromatic dispersion tolerances [1]. However, phase continuity would offer even better spectral efficiency, such as Minimum Shift Keying (MSK). It is a representative of continuous phase modulation (CPM). And it holds the characteristics of constant envelop, continuous phase, compact power spectrum and fast roll-of side-lobes [2]. In [3, 4], it is focused on the transmitter by using specially modulators to generate optical two-amplitude minimum shift keying (2AMSK) signal. In [5-7], 2AMSK was proposed in the optical OFDM system.

In this paper, we propose a a novel digital optical communication system based on 2AMSK signal transmission with coherent detection. It can obtain a higher spectral efficiency and reduce the complexity of the transmitter. Meanwhile, 100-Gb/s 2AMSK signal is generated and transmitted over 10 spans of $40 \mathrm{~km}$ standard single mode fiber (SSMF) and $8 \mathrm{~km}$ dispersion compensation fiber (DCF).

\section{Principles}

\section{A. The principle of 2AMSK signal}

MSK can be viewed as a special type of continuous phase-frequency shift keying (CPFSK) with modulation index $\mathrm{h}=0.5$, it can be expressed as [5]:

$$
S_{M S K}=\cos \left(\omega_{c} t+\frac{\pi a_{k}}{2 T_{b}}+\varphi_{k}\right)
$$

Where $a_{k}= \pm 1 . \varphi_{k}$ is the initial phase .

The 2AMSK signal is obtained by superposition of two MSK signals with different amplitudes. The amplitudes of the constituent signals are not equal, thus it can prevent the phase trajectory from passing through the origin. The amplitude ratio (AR) of the constituent signals can be defined as $\mathrm{AR}=\mathrm{b} / \mathrm{a}$, $\mathrm{a}$ and $\mathrm{b}$ are radius of constituent signals. In this way, the 2AMSK signal can be given by: 


$$
S_{2 A M S K}=A(t)\left[I_{k} \cos \left(\frac{\pi t}{2 T_{b}}\right) \cos \left(\omega_{c} t\right)-Q_{k} \sin \left(\frac{\pi t}{2 T_{b}}\right) \sin \left(\omega_{c} t\right)\right]
$$

Where $I_{k}=\cos \varphi_{k}, Q_{k}=a_{k} \cos \varphi_{k} . \mathrm{A}(\mathrm{t})=\mathrm{a}, \mathrm{b}(\mathrm{a} \neq \mathrm{b})$, it represent the amplitude information.

\section{$B$. The structure of the coherent optical 2AMSK system}

The structure of the proposed optical 2AMSK system is shown in Figure.1. It consists of 2AMSK transmitter, optical quadrature modulator, fiber link and 2AMSK receiver. At the transmitter, pseudo random binary sequences (PRBS) are differential encoded and split into two parts by serial-to-parallel mapping. Then the resulting half-rate bit streams are multiplied with sinusoidal signals of quarter bit frequency, thus the baseband MSK signal in electrical field is generated. And the baseband MSK signal is sent into amplitude modulation (AM). In addition, A(t) present the amplitude signals. Meanwhile, it has two different amplitudes so as to make the four phases switching between two ranges. In this way, the baseband 2AMSK signal can be generated to form the driving signals. A continuous wave (CW) light is generated from a commercial external cavity laser (ECL) source. And the baseband 2AMSK signal is modulated onto an optical carrier with quadrature modulator (QM), which consists of two arms. The upper includes a 1 bit delay and a Mach-Zehnder modulator (MZM1), while the lower has another MZM (MZM2) and a phase shifter to make the two arms have 90 degree phase deviation. After the QM, the generated optical 2AMSK signal is transmitted over fiber link. Before the fiber link, an erbium-doped fiber amplifier (EDFA1) is used to control the fiber launch power. The fiber link is composed of standard single mode fiber (SSMF) and dispersion compensating fiber (DCF) which fully compensates for the chromatic dispersion of the SSMF. Subsequently, an additional EDFA2 compensates for the loss of the loop switch and keeps the loop gain uniformly. The noise loading circuit is composed of an EDFA3 and a variable attenuator (VA). It is used to adjust the optical signal to noise ratio (OSNR) to the desired level.

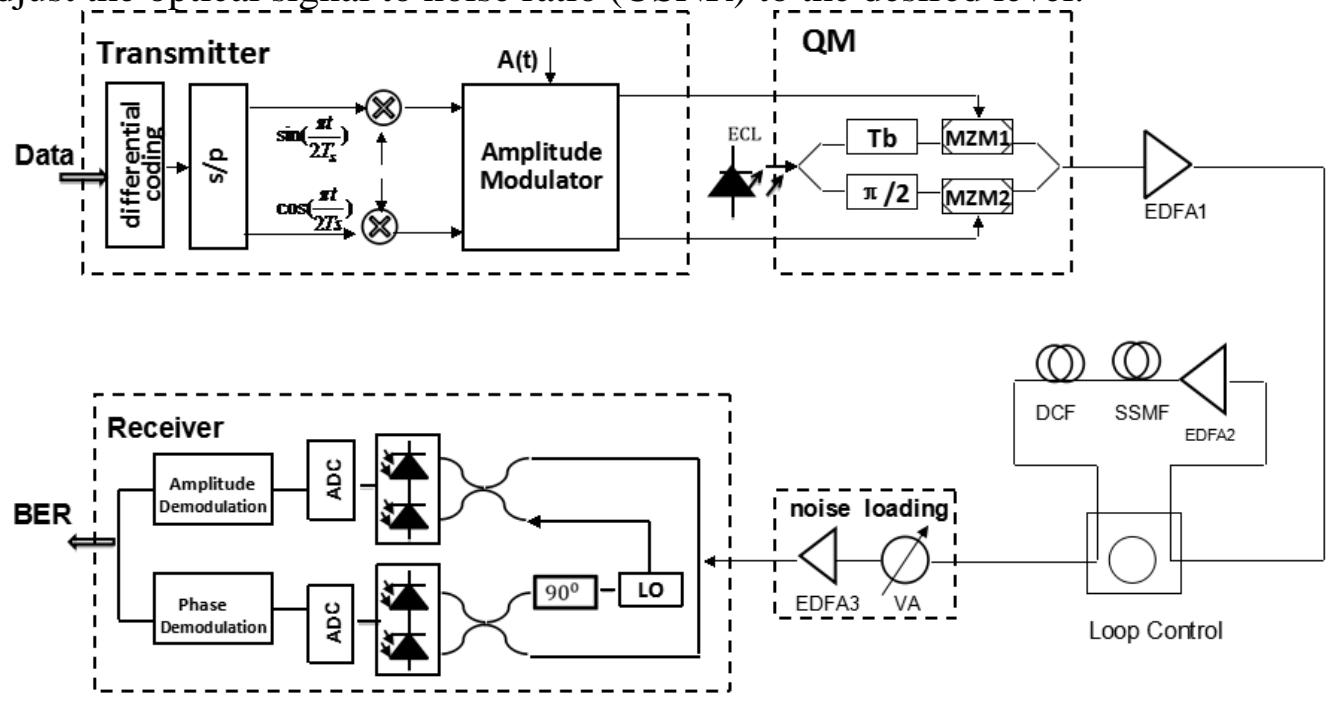

Fig.1. The structure of the optical 2AMSK system.

At the receiver, the optical 2AMSK signal is coupled with the local oscillator (LO) by the 90-degree hybrid coupler. With the different phase shift for the In-phase and Quadrature components, the phase information in the 2AMSK signal can be conversed to the intensity information. Then the original data can be recovered with appropriate thresholds setting in I and Q components, respectively, down-converted and stored for processing by off-line MATLAB. The electrical signal receiver consists of amplitude and phase detections. It extract the amplitude information by setting a threshold, and orthogonal differential demodulation method is used to detect the phase information.

\section{Simulation}

At the transmitter, a continuous wave (CW) light is generated from a commercial external cavity laser (ECL) source at the wavelength of $1550 \mathrm{~nm}$ without laser line-width. The bit rate of signal is $100-\mathrm{Gb} / \mathrm{s}$ and the laser frequency is $193.1 \mathrm{THz}$. The CW light is injected into a quadrature modulator, 
which is driven by the 2AMSK signal. The input data length is 16384, the sampling rate is 2 and the AR is set to be $0.25 / 0.75$. MZM1 and MZM2 are biased at the null point. They can driven by two data streams with a driving voltage of $2 \mathrm{~V}_{\pi}$, respectively. The driving voltage is about $8 \mathrm{~V}$. Before launched into the optical fiber, the 2AMSK signal is amplified by a commercial EDFA1 incorporated with a gain of $20 \mathrm{~dB}$ and a noise figure of $4 \mathrm{~dB}$. It can control the launch power of $2 \mathrm{dBm}$. The gain of EDFA2 and EDFA3 are $8 \mathrm{~dB}$ and $10 \mathrm{~dB}$, respectively. And the noise figure of them is $2 \mathrm{~dB}$. The output optical LDPC code 2AMSK signal is then sent into the fiber link. The fiber link consists of 10 spans of $48 \mathrm{~km}$ cascade fibers (40 km SSMF with $16 \mathrm{ps} / \mathrm{nm} / \mathrm{km}$ and $8 \mathrm{~km} \mathrm{DCF}$ with $80 \mathrm{ps} / \mathrm{nm} / \mathrm{km}$ ). After optical fiber transmission, the received signal is fed into $\mathrm{O} / \mathrm{E}$ conversion. The constellation trajectory of the 2AMSK signal before and after transmission are shown in Fig.2. Due to the amplified spontaneous emission (ASE) noises from the amplifiers and inevitable residual dispersion, the constellation diagram have certain diffusion.

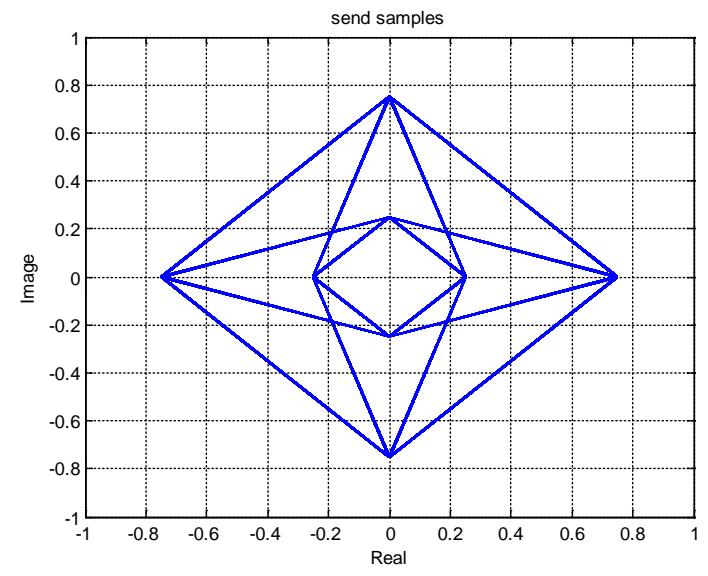

(a)

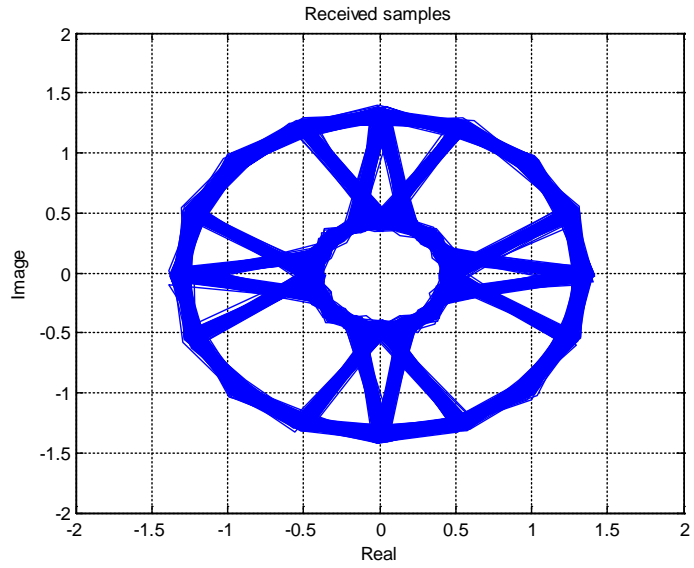

(b)

Fig.2. The constellation trajectory of the 2AMSK before and after transmission

Figure.3 compares the simulated power spectra of 100-Gb/s optical 2AMSK, 200-Gb/s optical 4AMSK, 100-Gb/s optical 2ADQPSK and 100-Gb/s optical 4ADPSK signals. For better analyze the band features, we set the resolution of $0.01 \mathrm{~nm}$ in the simulation. The power spectrum of optical 100-Gb/s 2AMSK format has similar characteristics to that of the 200-Gb/s 4AMSK counterpart, and the spectrum of $100-\mathrm{Gb} / \mathrm{s}$ 2ADQPSK signals is close to $100-\mathrm{Gb} / \mathrm{s}$ 4ADPSK, but the proposed 2AMSK offers narrowest spectral width and lowest side-lopes. This helps to obtain higher spectral efficiency, larger dispersion tolerance and reduces the effects such as inter-carrier interference (ICI) .

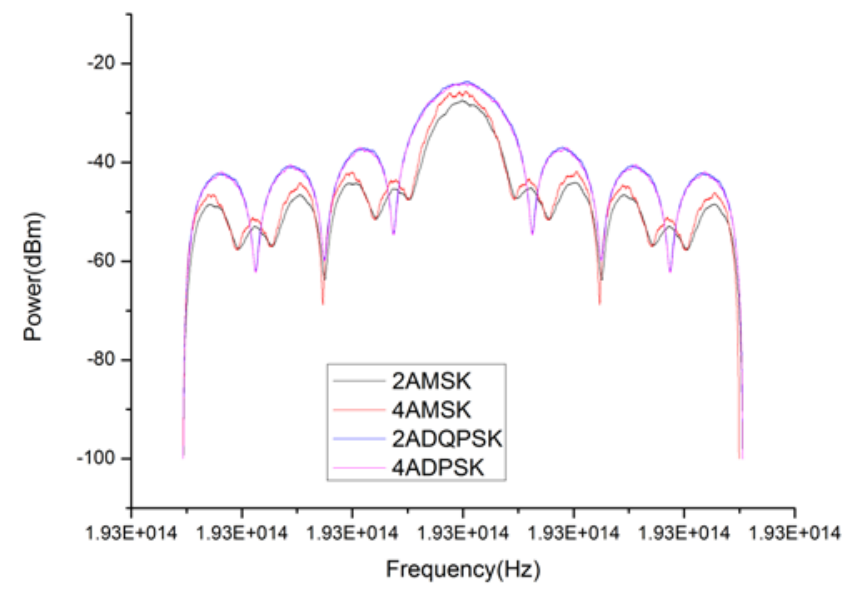

Fig.3. Spectra of four different schemes

Figure.4 shows the relationship between the measured BER and the OSNR of the three modulation formats. The data rate is $100-\mathrm{Gb} / \mathrm{s}$, and the transmission distance is set as 10 loop, namely $480 \mathrm{~km}$. As can be seen from the Fig.4, the system performance is substantially determined by the OSNR. 
Compared with 2ADQPSK and 4ADPSK, at a BER of $1 \times 10^{-3}$, the OSNR penalty of the 2AMSK signal is improved about $1.5 \mathrm{~dB}$ and $8 \mathrm{~dB}$, respectively. In fact, the 2AMSK format has continuous phase, which can effectively reduce the signal bandwidth. Therefore, the 2AMSK modulation format has greater dispersion tolerance than that of the other two modulation formats.

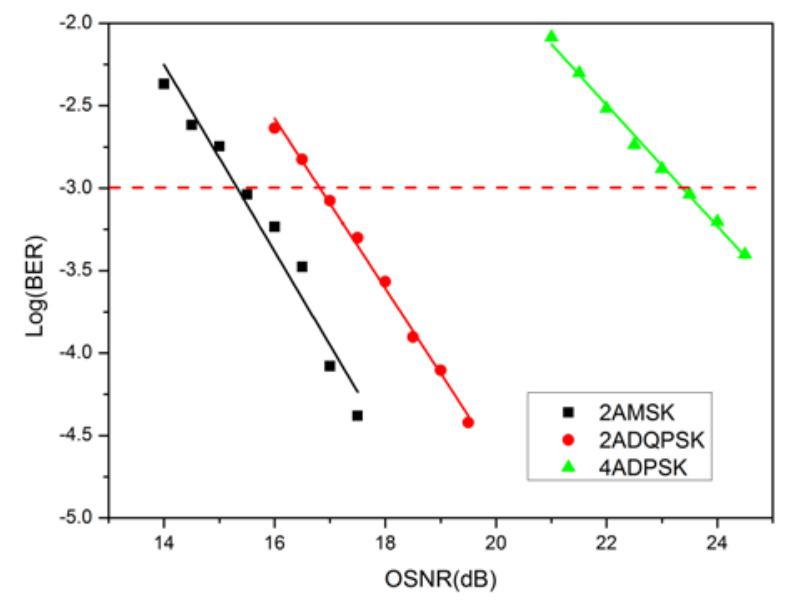

Fig.4. The measured BER curves OSNR in different schemes

\section{Conclusion}

In the paper, we have proposed a digital optical communication system based on optical two-amplitude minimum shift keying (2AMSK) signal transmission with coherent detection is proposed. 100-Gb/s optical 2AMSK signal has been transmitted over 10 spans of $40 \mathrm{~km} \mathrm{SSMF}$ and 8 $\mathrm{km}$ DCF. The simulation results show that, compared with 2ADQPSK and 4ADPSK, the proposed 2AMSK modulation format offers narrowest spectral width and lowest side-lopes. Moreover, the OSNR penalty of the 2AMSK signal is improved about $1.5 \mathrm{~dB}$ and $8 \mathrm{~dB}$, respectively.

\section{Acknowledgement}

This work is supported by the National Natural Science Foundation of China (61307087, 61377079), by the National "863" High Tech Research and Development Program of China (2011AA010203), and by the Fundamental Research Funds for the Central Universities and Young Teachers Program of Hunan University.

\section{References}

[1] A.Y. Yang, Y. Xiao and Y. Sun: Optical Fiber Communication \& Optoelectronic Exposition \& Conference (Shanghai, China, October, 2006). p.1-3.

[2] J.Y. Mo, Y. Dong, Y. J. Wen, S. Takahashi, X.Y. Wang and C. Lu: European Conference On Optical Communication (European, September 25-29,2005).Vol. 4, p.781-782.

[3] L. N. Binh and T.L. Huynh: Optics Communications, Vol. 281 (2008) No.17, p.4245-4253.

[4] L. N. Binh and T.L. Huynh: Lightwave Technology, Vol. 27 (2009) No.5, p.522-537.

[5] A.A. Eltholth, A.R. Mikhail, A. Elsherbini, Dessouky, M.I and A.I. Abdelfattah: EUROCON (Warsaw, Poland, September 9-12, 2007). Vol. 1, p.1057-1060.

[6] B.Liu, X. Xin ,L. Zhang, C.X. Yu and Y.J. Wang: Photonics Technology Letters (Los Angeles, CA, March 6-10, 2011). Vol. 23, p.1.

[7] L.J.Zhang: Photonics Technology Letters (Februray 15, 2013).Vol. 25, p.397-400. 